\title{
Identification and Enumeration of Small-Spored Alternaria Species Associated with Potato in the U.S. Northwest
}

\author{
Lydia S. Tymon, Tobin L. Peever, and Dennis A. Johnson, Washington State University, Department of Plant Pathology, Pullman 99164
}

\begin{abstract}
Tymon, L. S., Peever, T. L., and Johnson, D. A. 2016. Identification and enumeration of small-spored Alternaria species associated with potato in the U.S. Northwest. Plant Dis. 100:465-472.

A number of Alternaria spp. have been isolated from potato worldwide but only Alternaria solani and A. alternata have been described as pathogenic to this host in the United States. These taxa are easily differentiated based on conidial morphology but species delimitation among the small-spored Alternaria spp. associated with potato are much more challenging. Accurate identification methods for smallspored Alternaria spp. are necessary so that a more thorough understanding of Alternaria epidemiology can be obtained. Isolations of Alternaria fungi from lesions on potato leaves collected in the U.S. Northwest were made between 2008 and 2011. Large-spored taxa (putatively A. solani), were isolated less frequently than small-spored taxa (putatively A. alternata sensu lato), except in 2010. Colletotrichum coccodes was isolated from necrotic lesions in 2008 to 2010 but not in 2011. Frequency of isolation ranged from $0.05(5 \%)$ to $0.11(11 \%)$ during the 3 years the fungus was detected. Anonymous genomic region

OPA1-3, previously used for Alternaria systematics, allowed for the discrimination of phylogenetic lineages among 210 small-spored isolates. When OPA1-3 was restricted using enzyme ApaI, 65 isolates $(31 \%)$ displayed a restriction banding pattern consistent with previously characterized morphospecies A. alternata and A. tenuissima and 145 (69\%) displayed a restriction banding pattern consistent with the previously characterized morphospecies A. arborescens. Morphological characterization of a subsample of 59 small-spored Alternaria isolates randomly selected with each restriction pattern was compared with phylogenetic lineage. In all, $54(92 \%)$ isolates were consistently assigned to the same group by both methods. Three isolates exhibited conidial morphologies that were inconsistent with any described morphospecies. A small number of isolates were identified as $A$. arbusti (infectoria group) via sequencing of the glyceraldehyde-3-phosphate-dehydrogenase locus and BLAST searches.
\end{abstract}

A number of Alternaria spp. have been isolated from potato worldwide (Ardestani et al. 2010; Rodrigues et al. 2010; van der Waals et al. 2011) but only Alternaria solani and A. alternata have been described as pathogenic to this host in the United States. Early blight, caused by A. solani Sorauer 1896, is a major disease of potato in the U.S. Northwest that results in tan lesions with distinctive concentric rings on the foliage (Rotem 1994). Infection can reduce overall yields, tuber size, and tuber quality, and disease control practices can account for up to $10 \%$ of production costs (Stevenson et al. 2007). A. solani is recognized as the dominant pathogen infecting potato, although A. alternata (Fr.) Keissl. 1912 is increasingly affecting American potato production (Nolte 2008).

Infection by A. alternata, which causes brown spot, is characterized by small, irregular to circular lesions on leaves that can expand to approximately $3 \mathrm{~mm}^{2}$. These lesions can coalesce, resulting in significant leaf damage (Kirk and Wharton 2012). Brown spot can be difficult to diagnose in the field because symptoms overlap with those of early blight (Kirk and Wharton 2012) and recovery of A. alternata from lesions does not necessarily confirm brown spot disease. A. alternata can behave as a saprophyte (Rotem 1994) and A. alternata was not considered a pathogen of potato until 1984, when A. alternata was isolated from potato leaf lesions and tubers in Israel (Droby et al. 1984a,b). Inoculum concentration and disease incidence were positively correlated and infection of potato tubers by A. alternata resulted in sunken, black-pitted lesions (Droby et al. 1984b). Young lesions caused by A. alternata can also be confused

Corresponding author: L. S. Tymon; E-mail: lydia.tymon@email.wsu.edu

PPNS number 0636, Department of Plant Pathology, College of Agricultural, Human, and Natural Resource Sciences Agricultural Research Center, Hatch Project number 0678, Washington State University, Pullman 99164-6430.

Accepted for publication 17 August 2015.

http://dx.doi.org/10.1094/PDIS-03-15-0263-RE

(C) 2016 The American Phytopathological Society with lesions caused by Colletotrichum coccodes (Wallr.) S. Hughes 1958, which causes black dot (Mohan et al. 1992).

Species delimitation in Alternaria is challenging due to a paucity of morphological characteristics and considerable morphological plasticity (Rotem 1994; Simmons 2007). Simmons arranged the genus Alternaria into 14 morphospecies based on morphological features, primarily the three-dimensional sporulation apparatus (Simmons 1992). In general, morphospecies classifications have been concordant with clades defined through molecular systematic analyses (Hong et al. 2005; Peever et al. 2004; Pryor and Bigelow 2003; Pryor and Gilbertson 2000). However, some morphospecies have been associated with more than one clade or multiple morphospecies associated with the same clade (Andrew et al. 2009; Lawrence et al. 2013; Peever et al. 2004; Pryor and Michailides 2002). Although molecular sequence data have made species identification more accurate and facilitated the determination of evolutionary relationships among Alternaria spp., phylogenetic resolution is highly dependent upon loci used for analyses (Hong et al. 2005; Peever et al. 2004; Pryor and Bigelow 2003; Pryor and Gilbertson 2000). Species designation within the small-spored Alternaria taxa is further confounded by the inability of sequence data to distinguish between two morphologically distinct fungi, A. alternata and A. tenuissima (Andrew et al. 2009; Hong et al. 2005; Pryor and Michailides 2002). A comprehensive survey using both morphological and molecular criteria is required to assess the significance of different small-spored Alternaria taxa associated with potato in the United States.

Accurate identification of fungi is critical for disease management. For example, populations of A. solani and A. alternata differed in resistance to specific fungicides in the Columbia Basin (Tymon and Johnson 2014), which necessitates different choices of fungicides and rotations of fungicides. Three small-spored Alternaria spp. that are difficult to distinguish from $A$. alternata based on morphology are A. tenuissima, A. arborescens, and $A$. infectoria (Andersen and Thrane 1996; Taralova et al. 2011). However, A. tenuissima and A. infectoria can be distinguished from A. alternata and A. arborescens based on key morphological features (Simmons 2007). A. tenuissima 
Table 1. Species designation of small-spored Alternaria isolates from potato in the Pacific Northwest as Alternaria alternata or A. arborescens based on morphology and a restriction digest assay

\begin{tabular}{|c|c|c|c|c|}
\hline \multirow[b]{2}{*}{ Isolate } & \multirow[b]{2}{*}{ Location $^{\mathbf{a}}$} & \multicolumn{2}{|c|}{ Species designation } & \multirow[b]{2}{*}{ GenBank accession number } \\
\hline & & Morphology & Restriction digest $^{b}$ & \\
\hline aa10_216 & $\mathrm{CB}$ & alternata & alternata/tenuissima & $\ldots$ \\
\hline aa10_351 & SRV & alternata & alternata/tenuissima & $\ldots$ \\
\hline aa08_21 & $\mathrm{CB}$ & arborescens & alternata/tenuissima* & $\ldots$ \\
\hline aa10_253 & $\mathrm{BF}$ & arborescens & alternata/tenuissima* & $\ldots$ \\
\hline aa11_300 & SRV & arborescens & alternata/tenuissima* & $\ldots$ \\
\hline aa08_38 & $\mathrm{CB}$ & arborescens & arborescens & $\ldots$ \\
\hline aa09_12 & $\mathrm{CB}$ & arborescens & arborescens & $\ldots$ \\
\hline aa09_172 & $\mathrm{BF}$ & arborescens & arborescens & $\ldots$ \\
\hline aa09_60 & $\mathrm{CB}$ & arborescens & arborescens & $\ldots$ \\
\hline aa09_182 & $\mathrm{BF}$ & arborescens & arborescens & $\ldots$ \\
\hline aa09_225 & $\mathrm{CB}$ & arborescens & arborescens & $\ldots$ \\
\hline aa10_14 & $\mathrm{CB}$ & arborescens & arborescens & $\ldots$ \\
\hline aa10_20 & $\mathrm{CB}$ & arborescens & arborescens & $\ldots$ \\
\hline aa10_30 & $\mathrm{CB}$ & arborescens & arborescens & $\ldots$ \\
\hline aa10_32 & $\mathrm{CB}$ & arborescens & arborescens & $\ldots$ \\
\hline aa10_91 & $\mathrm{CB}$ & arborescens & arborescens & $\ldots$ \\
\hline aa10_105 & $\mathrm{CB}$ & arborescens & arborescens & $\ldots$ \\
\hline aa10_121 & SRV & arborescens & arborescens & $\ldots$ \\
\hline aa10_174 & $\mathrm{CB}$ & arborescens & arborescens & $\ldots$ \\
\hline aa10_207 & $\mathrm{CB}$ & arborescens & arborescens & $\ldots$ \\
\hline aa10_212 & $\mathrm{CB}$ & arborescens & arborescens & $\ldots$ \\
\hline aa10_280 & $\mathrm{BF}$ & arborescens & arborescens & $\cdots$ \\
\hline aa10_281 & SRV & arborescens & arborescens & $\ldots$ \\
\hline aa10_284 & SRV & arborescens & arborescens & $\ldots$ \\
\hline aa10_304 & $\mathrm{BF}$ & arborescens & arborescens & $\ldots$ \\
\hline aa10_310 & $\mathrm{BF}$ & arborescens & arborescens & $\ldots$ \\
\hline aa10_312 & SRV & arborescens & arborescens & $\ldots$ \\
\hline aa11_14 & OR & arborescens & arborescens & $\ldots$ \\
\hline aa11_20 & OR & arborescens & arborescens & $\ldots$ \\
\hline aa11_31 & $\mathrm{CB}$ & arborescens & arborescens & $\ldots$ \\
\hline aa11_41 & $\mathrm{CB}$ & arborescens & arborescens & $\ldots$ \\
\hline aa11_43 & $\mathrm{CB}$ & arborescens & arborescens & KM589065.1 \\
\hline aa11_45 & $\mathrm{CB}$ & arborescens & arborescens & $\ldots$ \\
\hline aa11_110 & $\mathrm{CB}$ & arborescens & arborescens & $\cdots$ \\
\hline aa11_115 & $\mathrm{CB}$ & arborescens & arborescens & $\ldots$ \\
\hline aa11_206 & $\mathrm{BF}$ & arborescens & arborescens & $\ldots$ \\
\hline aa11_234 & $\mathrm{CB}$ & arborescens & arborescens & $\ldots$ \\
\hline aa11_238 & $\mathrm{CB}$ & arborescens & arborescens & $\ldots$ \\
\hline aa11_253 & SRV & arborescens & arborescens & $\ldots$ \\
\hline aa11_257 & $\mathrm{BF}$ & arborescens & arborescens & $\ldots$ \\
\hline aa11_232 & $\mathrm{BF}$ & arborescens & arborescens & $\ldots$ \\
\hline aa11_352 & OR & arborescens & arborescens & $\ldots$ \\
\hline aa10_214 & $\mathrm{BF}$ & tenuissima & arborescens* & $\ldots$ \\
\hline aa11_290 & $\mathrm{BF}$ & tenuissima & arborescens* & $\ldots$ \\
\hline aa09_211 & $\mathrm{CB}$ & tenuissima & alternata/tenuissima & $\ldots$ \\
\hline aa10_21 & $\mathrm{CB}$ & tenuissima & alternata/tenuissima & $\ldots$ \\
\hline aa10_28 & $\mathrm{CB}$ & tenuissima & alternata/tenuissima & KM5890661.1 \\
\hline aa10_170 & $\mathrm{CB}$ & tenuissima & alternata/tenuissima & $\ldots$ \\
\hline aa10_180 & $\mathrm{CB}$ & tenuissima & alternata/tenuissima & $\ldots$ \\
\hline aa10_195 & $\mathrm{CB}$ & tenuissima & alternata/tenuissima & $\ldots$ \\
\hline aa10_198 & $\mathrm{BF}$ & tenuissima & alternata/tenuissima & $\ldots$ \\
\hline aa10_224 & $\mathrm{BF}$ & tenuissima & alternata/tenuissima & $\begin{array}{l}\cdots \\
\ldots\end{array}$ \\
\hline aa10_231 & $\mathrm{BF}$ & tenuissima & alternata/tenuissima & $\cdots$ \\
\hline aa10_263 & $\mathrm{CB}$ & tenuissima & alternata/tenuissima & $\ldots$ \\
\hline aa10_273 & $\mathrm{CB}$ & tenuissima & alternata/tenuissima & $\ldots$ \\
\hline aa10_317 & $\mathrm{BF}$ & tenuissima & alternata/tenuissima & \\
\hline aa11_09 & OR & tenuissima & alternata/tenuissima & $\ldots$ \\
\hline aa11_72 & $\mathrm{CB}$ & tenuissima & alternata/tenuissima & $\cdots$ \\
\hline aa11_229 & $\mathrm{CB}$ & tenuissima & alternata/tenuissima & $\begin{array}{l}\cdots \\
\cdots\end{array}$ \\
\hline aa08_33 & $\mathrm{CB}$ & $\ldots$ & alternata/tenuissima & $\ldots$ \\
\hline \multirow[t]{2}{*}{ aa08_37 } & $\mathrm{CB}$ & $\cdots$ & alternata/tenuissima & $\ldots$ \\
\hline & & & & (continued on next page) \\
\hline
\end{tabular}

a Location of isolate collection: $\mathrm{CB}=$ Columbia Basin, WA; BF = Bonner's Ferry, ID; SRV = Snake River Valley, ID; OR = LaGrande, OR; MI = Three Rivers, $\mathrm{MI} ;$ and $\mathrm{CO}=$ Alamosa, $\mathrm{CO}$.

b An asterisk (*) indicates that species designation based upon morphology and restriction digest were inconsistent. DNA was reextracted from colonies used in the morphological assay and restriction digest was performed a second time. Results from the two restriction digest assays were consistent. 
Table 1. (continued from preceding page)

\begin{tabular}{|c|c|c|c|c|}
\hline \multirow[b]{2}{*}{ Isolate } & \multirow[b]{2}{*}{ Location $^{\mathbf{a}}$} & \multicolumn{2}{|c|}{ Species designation } & \multirow[b]{2}{*}{ GenBank accession number } \\
\hline & & Morphology & Restriction digest $^{\mathbf{b}}$ & \\
\hline aa09_63 & $\mathrm{CB}$ & $\ldots$ & alternata/tenuissima & $\ldots$ \\
\hline aa10_36 & $\mathrm{CB}$ & $\ldots$ & alternata/tenuissima & $\ldots$ \\
\hline aa10_43 & $\mathrm{CB}$ & $\ldots$ & alternata/tenuissima & $\ldots$ \\
\hline aa10_49 & $\mathrm{CB}$ & $\ldots$ & alternata/tenuissima & $\ldots$ \\
\hline aa10_50 & $\mathrm{CB}$ & $\ldots$ & alternata/tenuissima & $\ldots$ \\
\hline aa10_88 & $\mathrm{CB}$ & $\ldots$ & alternata/tenuissima & $\ldots$ \\
\hline aa10_103 & $\mathrm{CB}$ & $\ldots$ & alternata/tenuissima & $\ldots$ \\
\hline aa10_104 & $\mathrm{CB}$ & $\ldots$ & alternata/tenuissima & $\ldots$ \\
\hline aa10_160 & $\mathrm{CB}$ & $\ldots$ & alternata/tenuissima & $\ldots$ \\
\hline aa10_184 & $\mathrm{CB}$ & $\ldots$ & alternata/tenuissima & $\ldots$ \\
\hline aa10_193 & $\mathrm{CB}$ & $\ldots$ & alternata/tenuissima & $\ldots$ \\
\hline aa10_252 & $\mathrm{BF}$ & $\ldots$ & alternata/tenuissima & $\ldots$ \\
\hline aa10_270 & $\mathrm{BF}$ & $\ldots$ & alternata/tenuissima & $\ldots$ \\
\hline aa10_313 & $\mathrm{BF}$ & $\ldots$ & alternata/tenuissima & $\ldots$ \\
\hline aa10_327 & $\mathrm{BF}$ & $\ldots$ & alternata/tenuissima & KM5 89063.1 \\
\hline aa10_345 & $\mathrm{BF}$ & $\ldots$ & alternata/tenuissima & $\ldots$ \\
\hline aa11_13 & $\mathrm{CB}$ & $\ldots$ & alternata/tenuissima & $\cdots$ \\
\hline aa11_21 & OR & $\ldots$ & alternata/tenuissima & $\ldots$ \\
\hline aa11_28 & $\mathrm{CB}$ & $\ldots$ & alternata/tenuissima & $\ldots$ \\
\hline aa11_33 & $\mathrm{CB}$ & $\ldots$ & alternata/tenuissima & $\ldots$ \\
\hline aa11_37 & $\mathrm{CB}$ & $\ldots$ & alternata/tenuissima & $\ldots$ \\
\hline aa11_42 & $\mathrm{CB}$ & $\ldots$ & alternata/tenuissima & $\ldots$ \\
\hline aa11_47 & $\mathrm{CB}$ & $\ldots$ & alternata/tenuissima & $\ldots$ \\
\hline aa11_114 & $\mathrm{CB}$ & $\ldots$ & alternata/tenuissima & $\ldots$ \\
\hline aa11_188 & $\mathrm{BF}$ & $\ldots$ & alternata/tenuissima & $\ldots$ \\
\hline aa11_245 & $\mathrm{BF}$ & $\ldots$ & alternata/tenuissima & $\ldots$ \\
\hline aa11_251 & $\mathrm{BF}$ & $\ldots$ & alternata/tenuissima & $\ldots$ \\
\hline aa11_282 & $\mathrm{CB}$ & $\ldots$ & alternata/tenuissima & $\ldots$ \\
\hline aa11_298 & $\mathrm{CB}$ & $\ldots$ & alternata/tenuissima & $\ldots$ \\
\hline aa11_299 & $\mathrm{CB}$ & $\ldots$ & alternata/tenuissima & $\ldots$ \\
\hline aa11_301 & $\mathrm{BF}$ & $\ldots$ & alternata/tenuissima & $\ldots$ \\
\hline aa11_317 & SRV & $\ldots$ & alternata/tenuissima & $\ldots$ \\
\hline aa11_329 & MI & $\ldots$ & alternata/tenuissima & $\ldots$ \\
\hline aa11_332 & MI & $\ldots$ & alternata/tenuissima & $\ldots$ \\
\hline aa11_337 & OR & $\ldots$ & alternata/tenuissima & $\ldots$ \\
\hline aa11_342 & OR & $\ldots$ & alternata/tenuissima & $\ldots$ \\
\hline aa11_348 & MI & $\ldots$ & alternata/tenuissima & $\ldots$ \\
\hline Othello_2011 & $\mathrm{CB}$ & $\ldots$ & alternata/tenuissima & $\ldots$ \\
\hline r011_r25e & OR & $\ldots$ & alternata/tenuissima & $\ldots$ \\
\hline ro11_r19a & $\mathrm{CB}$ & $\ldots$ & alternata/tenuissima & $\ldots$ \\
\hline ro11_r38d & OR & $\ldots$ & alternata/tenuissima & $\ldots$ \\
\hline ro11_r4_R & OR & $\ldots$ & alternata/tenuissima & $\ldots$ \\
\hline ro11_r4B & OR & $\ldots$ & alternata/tenuissima & $\ldots$ \\
\hline aa09_04 & $\mathrm{CB}$ & $\ldots$ & arborescens & $\ldots$ \\
\hline aa09_28 & $\mathrm{BF}$ & $\ldots$ & arborescens & $\ldots$ \\
\hline aa09_50 & $\mathrm{CB}$ & $\ldots$ & arborescens & $\ldots$ \\
\hline aa09_54 & $\mathrm{CB}$ & $\ldots$ & arborescens & $\ldots$ \\
\hline aa09_61 & $\mathrm{CB}$ & $\ldots$ & arborescens & $\ldots$ \\
\hline aa09_62 & $\mathrm{CB}$ & $\ldots$ & arborescens & $\ldots$ \\
\hline aa09_63 & $\mathrm{CB}$ & $\ldots$ & arborescens & $\ldots$ \\
\hline aa09_64 & $\mathrm{CB}$ & $\ldots$ & arborescens & $\ldots$ \\
\hline aa09_75 & $\mathrm{CB}$ & $\ldots$ & arborescens & $\ldots$ \\
\hline aa09_76 & $\mathrm{CB}$ & $\ldots$ & arborescens & $\ldots$ \\
\hline aa09_131 & $\mathrm{CB}$ & $\ldots$ & arborescens & $\ldots$ \\
\hline aa09_148 & $\mathrm{CB}$ & $\ldots$ & arborescens & $\ldots$ \\
\hline aa09_167 & $\mathrm{CB}$ & $\ldots$ & arborescens & $\ldots$ \\
\hline aa09_171 & $\mathrm{BF}$ & $\ldots$ & arborescens & $\ldots$ \\
\hline aa09_192 & $\mathrm{BF}$ & $\ldots$ & arborescens & $\ldots$ \\
\hline aa09_220 & $\mathrm{CB}$ & $\ldots$ & arborescens & $\ldots$ \\
\hline aa10_05 & $\mathrm{CB}$ & $\ldots$ & arborescens & $\ldots$ \\
\hline aa10_06 & $\mathrm{CB}$ & $\ldots$ & arborescens & $\ldots$ \\
\hline aa10_07 & $\mathrm{CB}$ & $\ldots$ & arborescens & $\ldots$ \\
\hline aa10_08 & $\mathrm{CB}$ & $\ldots$ & arborescens & $\ldots$ \\
\hline aa10_10 & $\mathrm{CB}$ & $\ldots$ & arborescens & $\ldots$ \\
\hline aa10_12 & $\mathrm{CB}$ & $\ldots$ & arborescens & $\ldots$ \\
\hline & & & & (continued on next page) \\
\hline
\end{tabular}


Table 1. (continued from preceding page)

\begin{tabular}{|c|c|c|c|c|}
\hline \multirow[b]{2}{*}{ Isolate } & \multirow[b]{2}{*}{ Location $^{\mathbf{a}}$} & \multicolumn{2}{|c|}{ Species designation } & \multirow[b]{2}{*}{ GenBank accession number } \\
\hline & & Morphology & Restriction digest $^{\mathbf{b}}$ & \\
\hline aa10_19 & $\mathrm{CB}$ & $\ldots$ & arborescens & $\ldots$ \\
\hline aa10_27 & $\mathrm{CB}$ & $\ldots$ & arborescens & $\ldots$ \\
\hline aa10_36 & $\mathrm{CB}$ & $\ldots$ & arborescens & $\ldots$ \\
\hline aa10_37 & $\mathrm{CB}$ & $\ldots$ & arborescens & $\ldots$ \\
\hline aa10_44 & $\mathrm{CB}$ & $\ldots$ & arborescens & $\ldots$ \\
\hline aa10_45 & $\mathrm{CB}$ & $\ldots$ & arborescens & $\ldots$ \\
\hline aa10_46 & $\mathrm{CB}$ & $\ldots$ & arborescens & $\ldots$ \\
\hline aa10_48 & $\mathrm{CB}$ & $\ldots$ & arborescens & $\ldots$ \\
\hline aa10_69 & $\mathrm{CB}$ & $\ldots$ & arborescens & $\cdots$ \\
\hline aa10_107 & $\mathrm{CB}$ & $\ldots$ & arborescens & $\cdots$ \\
\hline aa10_146 & $\mathrm{CB}$ & $\ldots$ & arborescens & $\ldots$ \\
\hline aa10_157 & SRV & $\ldots$ & arborescens & $\ldots$ \\
\hline aa10_160 & $\mathrm{CB}$ & $\ldots$ & arborescens & $\ldots$ \\
\hline aa10_172 & $\mathrm{CB}$ & $\ldots$ & arborescens & $\ldots$ \\
\hline aa10_199 & BF & $\ldots$ & arborescens & $\ldots$ \\
\hline aa10_203 & $\mathrm{CB}$ & $\ldots$ & arborescens & $\ldots$ \\
\hline aa10_205 & $\mathrm{CB}$ & $\ldots$ & arborescens & $\ldots$ \\
\hline aa10_206 & $\mathrm{BF}$ & $\ldots$ & arborescens & $\ldots$ \\
\hline aa10_209 & $\mathrm{BF}$ & $\ldots$ & arborescens & $\ldots$ \\
\hline aa10_210 & $\mathrm{BF}$ & $\ldots$ & arborescens & $\ldots$ \\
\hline aa10_22 & $\mathrm{CB}$ & $\ldots$ & arborescens & $\ldots$ \\
\hline aa10_223 & SRB & $\ldots$ & arborescens & $\ldots$ \\
\hline aa10_225 & $\mathrm{BF}$ & $\ldots$ & arborescens & $\ldots$ \\
\hline aa10_228 & $\mathrm{BF}$ & $\ldots$ & arborescens & $\ldots$ \\
\hline aa10_235 & $\mathrm{BF}$ & $\ldots$ & arborescens & $\ldots$ \\
\hline aa10_236 & $\mathrm{CB}$ & $\ldots$ & arborescens & $\ldots$ \\
\hline aa10_239 & $\mathrm{CB}$ & $\ldots$ & arborescens & $\ldots$ \\
\hline aa10_243 & $\mathrm{CB}$ & $\ldots$ & arborescens & $\ldots$ \\
\hline aa10_300 & $\mathrm{BF}$ & $\ldots$ & arborescens & $\ldots$ \\
\hline aa10_305 & $\mathrm{BF}$ & $\ldots$ & arborescens & $\ldots$ \\
\hline aa10_306 & $\mathrm{BF}$ & $\ldots$ & arborescens & $\ldots$ \\
\hline aa10_307 & $\mathrm{BF}$ & $\ldots$ & arborescens & KM589064.1 \\
\hline aa10_314 & $\mathrm{BF}$ & $\ldots$ & arborescens & $\ldots$ \\
\hline aa10_328 & $\mathrm{BF}$ & $\ldots$ & arborescens & $\ldots$ \\
\hline aa10_335 & SRV & $\ldots$ & arborescens & $\ldots$ \\
\hline aa10_344 & $\mathrm{BF}$ & $\ldots$ & arborescens & $\ldots$ \\
\hline aa10_347 & $\mathrm{BF}$ & $\ldots$ & arborescens & $\ldots$ \\
\hline aa10_348 & $\mathrm{BF}$ & $\ldots$ & arborescens & $\ldots$ \\
\hline aa10_350 & $\mathrm{BF}$ & $\ldots$ & arborescens & $\ldots$ \\
\hline aa10_352 & $\mathrm{CB}$ & $\ldots$ & arborescens & $\ldots$ \\
\hline aa11_16 & $\mathrm{CB}$ & $\ldots$ & arborescens & $\ldots$ \\
\hline aa11_32 & $\mathrm{CB}$ & $\ldots$ & arborescens & $\ldots$ \\
\hline aa11_34 & $\mathrm{CB}$ & $\ldots$ & arborescens & $\ldots$ \\
\hline aa11_39 & $\mathrm{CB}$ & $\ldots$ & arborescens & $\ldots$ \\
\hline aa11_40 & $\mathrm{CB}$ & $\ldots$ & arborescens & $\ldots$ \\
\hline aa11_44 & $\mathrm{CB}$ & $\ldots$ & arborescens & $\ldots$ \\
\hline aa11_49 & $\mathrm{CB}$ & $\ldots$ & arborescens & $\ldots$ \\
\hline aa11_59 & $\mathrm{CB}$ & $\ldots$ & arborescens & $\ldots$ \\
\hline aa11_77 & $\mathrm{CB}$ & $\ldots$ & arborescens & $\ldots$ \\
\hline aa11_80 & $\mathrm{CB}$ & $\ldots$ & arborescens & $\ldots$ \\
\hline aa11_109 & $\mathrm{CB}$ & $\ldots$ & arborescens & $\ldots$ \\
\hline aa11_113 & $\mathrm{CB}$ & $\ldots$ & arborescens & $\ldots$ \\
\hline aa11_152 & $\mathrm{CB}$ & $\ldots$ & arborescens & $\ldots$ \\
\hline aa11_154 & $\mathrm{CB}$ & $\ldots$ & arborescens & $\ldots$ \\
\hline aa11_161 & $\mathrm{CB}$ & $\ldots$ & arborescens & KM589067.1 \\
\hline aa11_183 & SRV & $\ldots$ & arborescens & $\ldots$ \\
\hline aa11_188 & $\mathrm{BF}$ & $\ldots$ & arborescens & $\ldots$ \\
\hline aa11_205 & $\mathrm{BF}$ & $\ldots$ & arborescens & $\ldots$ \\
\hline aa11_225 & $\mathrm{CB}$ & $\ldots$ & arborescens & $\ldots$ \\
\hline aa11_229 & $\mathrm{CB}$ & $\ldots$ & arborescens & $\ldots$ \\
\hline aa11_244 & $\mathrm{BF}$ & $\ldots$ & arborescens & $\ldots$ \\
\hline aa11_246 & $\mathrm{BF}$ & $\ldots$ & arborescens & $\ldots$ \\
\hline aa11_251 & $\mathrm{BF}$ & $\ldots$ & arborescens & $\cdots$ \\
\hline aa11_259 & $\mathrm{BF}$ & $\ldots$ & arborescens & $\ldots$ \\
\hline aa11_29 & $\mathrm{CB}$ & $\ldots$ & arborescens & $\ldots$ \\
\hline & & & & (continued on next page) \\
\hline
\end{tabular}


Table 1. (continued from preceding page)

\begin{tabular}{|c|c|c|c|c|}
\hline \multirow[b]{2}{*}{ Isolate } & \multirow[b]{2}{*}{ Location $^{\mathbf{a}}$} & \multicolumn{2}{|c|}{ Species designation } & \multirow[b]{2}{*}{ GenBank accession number } \\
\hline & & Morphology & Restriction digest $^{\mathbf{b}}$ & \\
\hline aa11_296 & MT & $\ldots$ & arborescens & $\ldots$ \\
\hline aa11_297 & $\mathrm{CB}$ & $\ldots$ & arborescens & $\ldots$ \\
\hline aa11_302 & $\mathrm{BF}$ & $\ldots$ & arborescens & $\ldots$ \\
\hline aa11_303 & SRV & $\ldots$ & arborescens & $\ldots$ \\
\hline aa11_305 & SRV & $\ldots$ & arborescens & $\ldots$ \\
\hline aa11_307 & SRV & $\ldots$ & arborescens & $\ldots$ \\
\hline aa11_311 & SRV & $\ldots$ & arborescens & $\ldots$ \\
\hline aa11_313 & MI & $\ldots$ & arborescens & $\ldots$ \\
\hline aa11_314 & SRV & $\ldots$ & arborescens & $\ldots$ \\
\hline aa11_316 & SRV & $\ldots$ & arborescens & $\ldots$ \\
\hline aa11_318 & $\mathrm{CB}$ & $\ldots$ & arborescens & $\ldots$ \\
\hline aa11_320 & $\mathrm{BF}$ & $\ldots$ & arborescens & $\ldots$ \\
\hline aa11_323 & $\mathrm{CB}$ & $\ldots$ & arborescens & $\ldots$ \\
\hline aa11_330 & $\mathrm{CO}$ & $\ldots$ & arborescens & $\ldots$ \\
\hline aa11_338 & ND & $\ldots$ & arborescens & $\ldots$ \\
\hline aa11_343 & $\mathrm{CB}$ & $\ldots$ & arborescens & $\ldots$ \\
\hline r011_r35a & OR & $\ldots$ & arborescens & $\ldots$ \\
\hline ro11_r17 & OR & $\ldots$ & arborescens & $\ldots$ \\
\hline ro11_r46B & OR & $\ldots$ & arborescens & $\ldots$ \\
\hline
\end{tabular}

produces conidia in long, unbranched chains whereas A. infectoria produces distinct secondary conidiophores (Simmons 2007). It is important to differentiate among these fungi because some small-spored species produce toxins that are involved in pathogenicity, while A. infectoria does not. AAL-toxin, produced by A. arborescens, affects mitochondria and rough endoplasmic reticulum of tomato (Rotem 1994), resulting in the formation of stem lesions as well as necrosis in leaf tissue (Gilchrist et al. 1995; Thomma 2003).

Small-spored Alternaria spp. associated with potato in the U.S. Northwest have been poorly studied and, therefore, are the primary focus of this study. Due to the number of small-spored Alternaria spp. that potentially can cause foliar lesions on potato, the objectives of this research were to (i) determine the frequency of different Alternaria taxa and C. coccodes associated with necrotic lesions on potato foliage in the U.S. Northwest and (ii) distinguish between A. alternata and the potential host-specific, toxin-producing A. arborescens. Discriminating among Alternaria spp. on potato in the U.S. Northwest will allow for future research on targeted management with fungicides and cultural tactics as well as providing further understanding of potential pathogen epidemiology.

\section{Materials and Methods}

Sampling and isolation. Potato fields in Washington and Idaho are usually managed with fungicides, which reduce the incidence of infection by Alternaria spp. When potato leaves with characteristic early blight and brown spot lesions were observed, leaves were collected. Leaves were collected by lab members, field managers, and Washington State extension agents from potato fields in the Columbia Basin of Washington and Oregon, the Skagit Valley of Washington, the Snake River Valley in Idaho, and near Bonner's Ferry, ID between 2008 and 2011 (Table 1). Foliage was placed in plastic bags, sealed, and transported to a laboratory in Pullman, WA. Leaves were stored at $4^{\circ} \mathrm{C}$ until processed. Potato leaflets with lesions were surface disinfested by dipping in $70 \%$ ethanol for 3 to $5 \mathrm{~s}$. Leaflets were blotted with paper towel and air dried. One to three lesions per leaf (one lesion per leaflet) were sampled. Lesion margins were excised and plated on modified potato dextrose agar (mPDA; Difco Bactoagar at $20 \mathrm{~g} / \mathrm{liter}$, Difco PDA at $1 \mathrm{~g} / \mathrm{liter}$, and $95 \%$ ethanol at $6 \mathrm{ml} /$ liter). Isolates were examined under a stereomicroscope and were assigned to A. solani, small-spored Alternaria, or $C$. coccodes based on morphological characteristics. Single conidial isolates were obtained by streaking a conidial suspension on mPDA. A single conidium was selected after $24 \mathrm{~h}$. Colonies were grown on mPDA at 21 to $23^{\circ} \mathrm{C}$ under continuous light. Isolates were grown on mPDA for 7 to 10 days and stored on sterile filter paper, as previously described (Peever et al. 1999), for future inoculation experiments. Filter paper was placed inside sterile coin envelopes and maintained at room temperature.

DNA extraction. Alternaria isolates were grown in Difco potato dextrose broth (Becton, Dickinson and Company, Franklin Lakes, $\mathrm{NJ}$ ) for 1 week and vacuum filtered, and mycelia were lyophilized for $48 \mathrm{~h}$. DNA was extracted using a modified phenol chloroform extraction (Dobinson 1995; Lee et al. 1990). Mycelia were vortexed in 2.0-ml microfuge tubes (Fisherbrand Premium; Fisher Scientific, Inc., Hampton, New Hampshire) with one ceramic bead (MP Biomedicals, LLC, Santa Ana, CA) until mycelia were ground to a fine powder. Lysis buffer $(200 \mu \mathrm{l}$ of $0.5 \mathrm{M} \mathrm{NaCl}, 0.2 \mathrm{M}$ Tris- $\mathrm{HCl}$ [pH 8.0], 0.01 M EDTA, and $1 \%$ sodium dodecyl sulfate) were added to the mixture. Powdered mycelia were then vigorously vortexed for $3 \mathrm{~min}$ at 6- to 30-s intervals, where cells were placed on ice every $30 \mathrm{~s}$. An additional $200 \mu \mathrm{l}$ of lysis buffer was added to the mycelia and briefly vortexed. Phenol-chloroform (300 $\mu 1,1: 1)$ was added and the sample was vortexed for $30 \mathrm{~s}$. The sample was centrifuged at $12,000 \mathrm{rpm}$ for $5 \mathrm{~min}$ to separate the phases. Phenol-chloroform $(300 \mu \mathrm{l})$ was added to the aqueous phase and the sample was vortexed for $30 \mathrm{~s}$. The aqueous phase was separated once more by centrifugation and a nucleic acid pellet was precipitated with $200 \mu$ l of $95 \%$ ethanol and placed on ice for $5 \mathrm{~min}$. This step was repeated without placing the sample on ice. The pellet was then washed with $200 \mu$ l of $70 \%$ ethanol. RNA was digested by $2 \mu \mathrm{l}$ of RNAse $(1 \mathrm{mg} / \mathrm{ml})$ to the DNA and incubated at $37^{\circ} \mathrm{C}$ overnight.

Polymerase chain reaction amplification and restriction digest assay. In all, 210 isolates were subsampled from 940 putative $A$. alternata and $A$. arborescens isolates for the restriction digest assay. Isolates with distinct secondary conidiophores were eliminated from this study because this is a characteristic of morphospecies A. infectoria (Simmons 2007) and beyond the scope of this assay. An anonymous 900-bp region, OPA1-3, was amplified using polymerase chain reaction (PCR) and digested with a restriction enzyme to identify A. arborescens (Peever et al. 2004). At this locus, $A$. alternata has two restriction sites, resulting in three bands, whereas isolates of $A$. arborescens have only one restriction site. PCR was performed using $25-\mu$ l reaction volumes in thin-walled $0.2-\mathrm{ml}$ PCR strip tubes. Reaction mixes comprised $2.0 \mu \mathrm{l}$ of $10 \times$ PCR buffer (NEB, Waltham, MA), $0.48 \mu \mathrm{M} \mathrm{MgCl} 2,2.4 \mu \mathrm{M}$ dNTP (Thermo Fisher Scientific, Waltham, MA), $0.1 \mu \mathrm{M}$ each primer (Invitrogen Life Technologies, Carlsbad, CA), $1 \mathrm{U}$ of Taq polymerase (NEB), and $20 \mathrm{ng}$ of DNA. Reactions were run using a 
touchdown PCR protocol on a Bio-Rad MyCycler Personal Thermal Cycler (Hercules, California). Thermal cycler conditions were 4 min at $94^{\circ} \mathrm{C}$ (denaturation) followed by 18 cycles of $45 \mathrm{~s}$ at $94^{\circ} \mathrm{C}$, $45 \mathrm{~s}$ at $61^{\circ} \mathrm{C}$ (with the temperature dropping $0.5^{\circ} \mathrm{C}$ each cycle), and $2 \mathrm{~min}$ at $72^{\circ} \mathrm{C}$; followed by 20 cycles of $45 \mathrm{~s}$ at $94^{\circ} \mathrm{C}, 45 \mathrm{~s}$ at $53^{\circ} \mathrm{C}$, and 2 min at $72^{\circ} \mathrm{C}$; with a final extension cycle of $10 \mathrm{~min}$ at $72^{\circ} \mathrm{C}$. Amplicons were run on a $1 \%$ agarose gel to confirm amplification. Amplicons were restricted with ApaI (Invitrogen Life Technologies) in $15-\mu l$ reaction volumes using $1 \mu l$ of enzyme and $2 \mu l$ of buffer per reaction. Samples were incubated at $37^{\circ} \mathrm{C}$ overnight and digested DNA was visualized on $1 \%$ agarose gels. Because a restriction digest of OPA1-3 cannot distinguish A. alternata from A. tenuissima, isolates were designated as either $A$. arborescens or A. alternata/tenuissima (Table 1). The OPA1-3 region of five representative isolates was amplified and sequenced to confirm identity of the isolates and integrity of the restriction digest. Sequences were aligned using Clustal $\mathrm{W}$ in Geneious 8.0 with reference sequences of A. alternata (GenBank accession number JQ859819.1), A. tenuissima (GenBank accession number JQ859820.1), and A. arborescens (GenBank accession number JQ859821.1). All reference sequences selected were from isolates identified by E. G. Simmons.

Sequencing. PCR cleanup was performed by adding $1.5 \mu \mathrm{l}$ of Exosap-It (Affymetrix, Santa Clara, CA) to $6 \mu \mathrm{l}$ of PCR product and incubating at $37^{\circ} \mathrm{C}$ for $15 \mathrm{~min}$ followed by an incubation at $80^{\circ} \mathrm{C}$ for $15 \mathrm{~min}$. DNA sequencing was performed by Elim Biopharm (Hayward, CA). BLAST searches were performed to the National Center for Biotechnology Information nucleotide sequence databases to identify the closest sequence matches.

Glyceraldehyde-3-phosphate-dehydrogenase amplification and sequencing. Three isolates exhibited conidial morphology that was inconsistent with A. alternata, A. tenuissima, or A. arborescens. Amplification of glyceraldehyde-3-phosphate-dehydrogenase (G3PD) was performed using gpd1 and 2 primers (Berbee et al. 1999) because the $G 3 P D$ locus is sufficient at distinguishing among different small-spored Alternaria groups (Pryor and Bigelow 2003). PCR was performed using $25-\mu \mathrm{l}$ reaction volumes in thin-walled $0.2-\mathrm{ml}$ PCR strip tubes. Reaction mixes comprised $2.0 \mu \mathrm{l}$ of $10 \times$ PCR buffer (NEB), $0.48 \mu \mathrm{M} \mathrm{MgCl}_{2}, 2.4 \mu \mathrm{M}$ dNTPs (Thermo Fisher Scientific), $0.1 \mu \mathrm{M}$ each primer (Invitrogen Life Technologies), 1 unit of Taq polymerase (NEB), and $20 \mathrm{ng}$ of DNA. Cycling was performed in a Techne TC-3000X Thermal Cycler (Burlington, NJ). For amplification of $G 3 P D$, cycles consisted of $4 \mathrm{~min}$ at $94^{\circ} \mathrm{C}$ followed by 35 cycles of $45 \mathrm{~s}$ at $94^{\circ} \mathrm{C}, 45 \mathrm{~s}$ at $57^{\circ} \mathrm{C}$, and $2 \mathrm{~min}$ at $72^{\circ} \mathrm{C}$, with a final extension cycle of $10 \mathrm{~min}$ at $72^{\circ} \mathrm{C}$.

Sequences were aligned using the Clustal $\mathrm{W}$ package (EMBL-EBI, Hixton, Cambridge, UK) in Geneious 8.0 (Biomatters, Auckland, NZ) with reference sequences of A. alternata (GenBank accession number AY278808.1), A. arborescens (GenBank accession number GQ180070.1), A. arbusti (GenBank accession number JQ693621), A. arbusti (GenBank accession number FJ214806.1), A. oregonensis (GenBank accession number AY762957.1), and A. metachromatica (GenBank accession number AY762956.1). All reference sequences selected were from isolates identified by E. G. Simmons.

Morphological characterization. Morphological characterization of isolates was conducted to estimate the stability of characteristics used to separate $A$. arborescens from A. alternata. A randomly selected subsample of 59 isolates identified as A. alternata or $A$. arborescens by the restriction digest assay were morphologically characterized on potato carrot agar, as prepared according to Simmons (2007). In all, $20 \mathrm{~g}$ of Yukon gold potato and $20 \mathrm{~g}$ of carrot were autoclaved in $100 \mathrm{ml}$ of distilled water for $30 \mathrm{~min}$. The potato and carrot were then sieved through a number 10 USA Standard Test Sieve (WS Tyler Industrial Group, Mentor, OH). The mashed potato and carrot were added to 1 liter of distilled $\mathrm{H}_{2} \mathrm{O}$ and $20 \mathrm{~g}$ of Difco BactoAgar (Becton, Dickinson and Company) and autoclaved for $20 \mathrm{~min}$. Autoclaved medium was cooled and poured into petri dishes (60 by 15 mm; Greiner, Frickenhausen, Germany).

In total, 59 individual isolates were plated in the center of a petri dish and maintained under a cycle of $8 \mathrm{~h}$ of fluorescent light and $16 \mathrm{~h}$ of darkness at a mean temperature of $24^{\circ} \mathrm{C}$ in light conditions

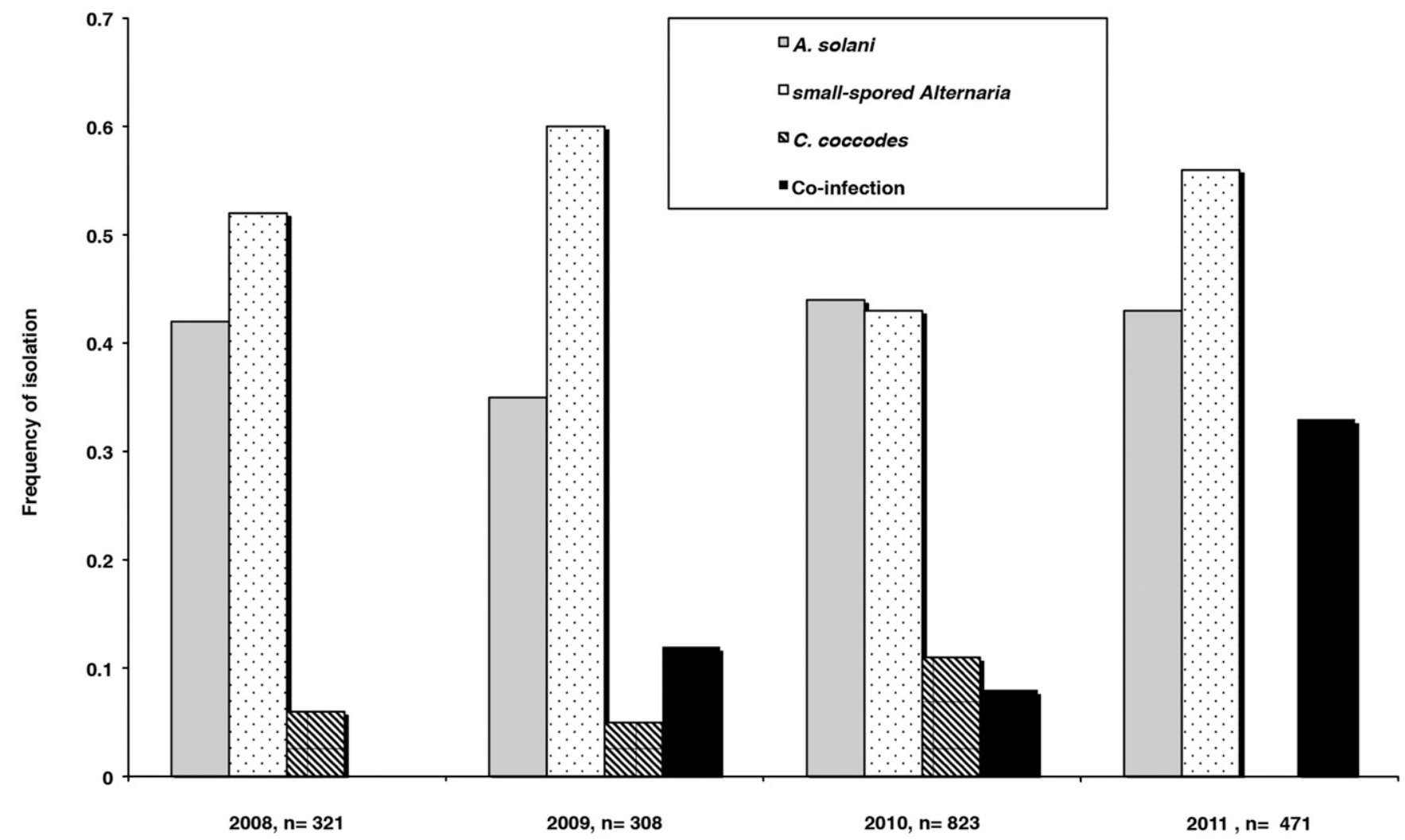

Fig. 1. Frequency of isolation of Alternaria spp. from early blight and brown spot lesions on potato in the U.S. Northwest. Frequency of isolation is given as a proportion of the total isolates collected in one year. 
and $23^{\circ} \mathrm{C}$ in dark conditions for 5 to 8 days. Fungi were mounted in lactic acid on slides using double-sided tape, where one side of the tape was touched gently to the fungal culture and the other side adhered to the slide. Conidia and conidiophores were examined with light microscopy on an Olympus $\mathrm{BH} 2$ compound microscope (Olympus Corp., Shinjuku, Tokyo) and photographed using a Nikon Coolpix 4300 (Nikon Corp., Tokyo) camera. Morphospecies assignments were based on descriptions of Alternaria spp. by Simmons (2007).

\section{Results}

Isolate collection and frequency of isolation. Small-spored Alternaria taxa were isolated more frequently than A. solani from leaves with lesions in all years except 2010. C. coccodes was isolated from necrotic lesions in 2008 to 2010 but not in 2011 (Fig. 1). Frequency of isolation ranged from $0.05(5 \%)$ to 0.11 (11\%) during the 3 years the fungus was detected. Data were not collected on coinfection of lesions with A. alternata and either A. solani or C. coccodes in 2008. Coinfection of lesions was observed in 2009 to 2011, although at infection frequencies of 0.33 or less.

OPA1-3 restriction digest, $G 3 P D$ amplification, and species identification. The anonymous region of randomly selected smallspored Alternaria isolates was amplified and restricted with ApaI. In all, $65(31 \%)$ of 210 isolates had a restriction-banding pattern that was consistent with $A$. alternata/tenuissima and $145(69 \%)$ had a restriction-banding pattern that was consistent with A. arborescens. Four isolates designated as A. alternata or A. arborescens by the restriction digest were confirmed with sequence data when the OPA1-3 region of six isolates was amplified and compared with sequences in GenBank.

Three isolates exhibited conidial morphology that was inconsistent with any described morphospecies. Sequencing of G3PD and BLAST searches identified these isolates as A. arbusti (KM589057.1, KM589056.1, and KM589054.1).

Morphological characterization. A. arbusti isolates (Fig. 2) were distinguishable from A. alternata and A. arborescens (Fig. 3) based on morphology. A. arbusti isolates produced aerial hyphae that gave the colony a cottony appearance. Conidia of $A$. arbusti are produced in short chains and, with the exception of the terminal conidium,
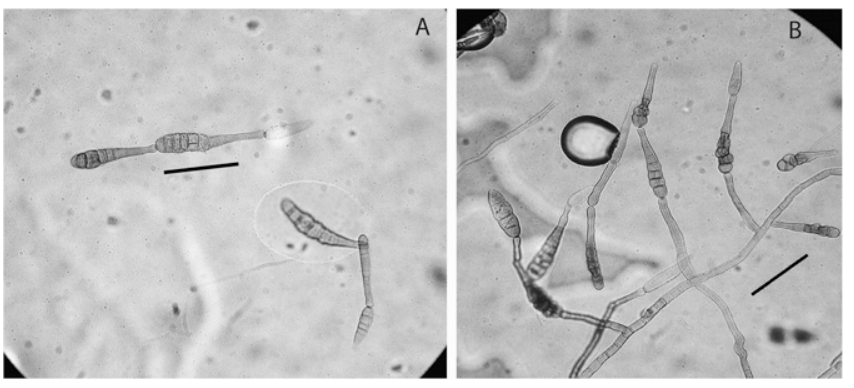

Fig. 2. Alternaria arbusti A, conidia and B, conidiophore. Scale bars $=34 \mu \mathrm{M}$. produce apical secondary conidiophores (Simmons 2007). Of 59 isolates, $2(3 \%)$ were putatively designated as morphospecies A. alternata, 40 (68\%) were designated as A. arborescens, and $17(29 \%)$ were designated as A. tenuissima based on branching pattern, conidial length and width, and conidiophore length (Table 1). However, the restriction digest assay determined that five isolate identifications were inconsistent between the morphological determination and restriction digest assay. Three isolates were designated as $A$. arborescens based on morphology but as A. alternata/tenuissima based on the restriction digest, and two isolates were designated as $A$. tenuissima based on the morphology but as A. arborescens based on the restriction digest. DNA was reextracted and the restriction digest assay was run to confirm that the inconsistencies observed were not due to isolate contamination.

\section{Discussion}

Morphology is the traditional means of distinguishing smallspored Alternaria spp. However, the morphological characteristics used to identify Alternaria spp. are plastic and susceptible to environmental variation, making species designation difficult. For example, A. brassicae forms conidia in chains when grown in pure culture but not when growing on the host (Rotem 1994). Substrate, temperature, and light can affect the dimensions of the spores, which often overlap among species, preventing accurate identification on the basis of spore dimensions. Lowered humidity or temperature during conidial development can increase conidial size (Misaghi et al. 1978). Five isolate identifications were inconsistent between the morphological determination and restriction digest assay. As with other species of Alternaria, A. arborescens morphology is plastic. When isolates are grown under the recommended conditions of light and darkness (Simmons 2007), conidiophores grown in lightexposed areas are shorter, up to 200 to $300 \mu \mathrm{m}$, whereas conidiophores grown in the dark are between $800 \mu \mathrm{m}$ and $1 \mathrm{~mm}$ or longer (Simmons 2007). This can lead to incorrectly identifying an A. arborescens isolate as A. alternata. Two isolates were designated as A. tenuissima based on the morphology but as A. arborescens based on the restriction digest. Further sequencing will be conducted to assess whether this inconsistency is due to morphological variation of the A. arborescens or if the isolate is a morphologically similar species to A. tenuissima.

The anonymous region OPA1-3 was one of three regions that has been shown to reliably distinguish among taxa of small-spored Alternaria spp., particularly within the alternata clade (Peever et al. 2004). The use of this restriction digest assay represented a robust means to distinguishing between morphospecies A. arborescens and A. alternata, particularly if key morphological features are not apparent. The assay is also rapid, allowing for many isolates to be assessed at one time, and is much more economical than sequencing. The accuracy of the assay was confirmed by sequencing of the OPA1-3 region.

Three isolates were identified as $A$. arbusti, which clusters within the infectoria clade (Lawrence et al. 2014). Along with being genetically distinct, past studies on fungal metabolite production by $A$. alternata and A. infectoria species show that metabolite profiles are also different
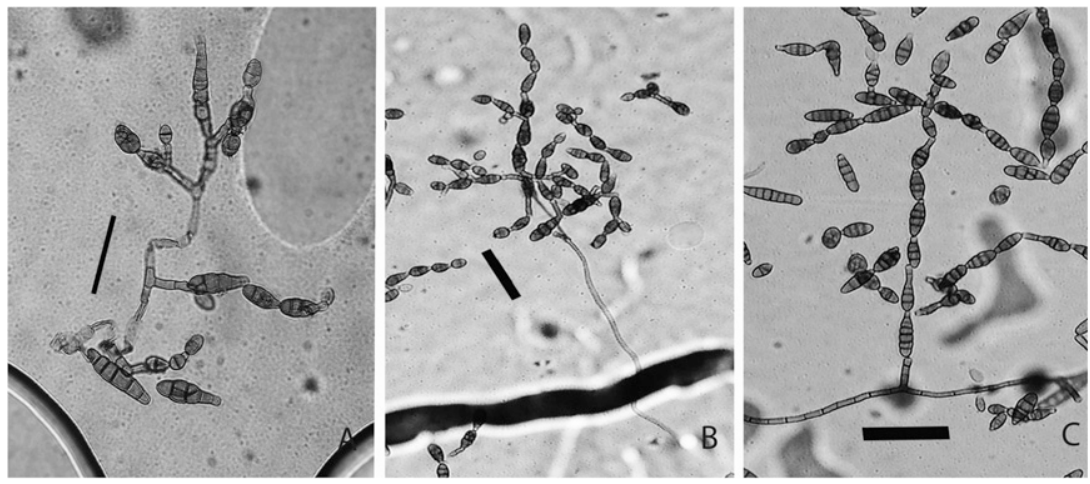

Fig. 3. Conidia and conidiophores of A, Alternaria alternata, scale $=35 \mu \mathrm{M} ; \mathrm{B}$, A. arborescens, scale $=53 \mu \mathrm{M}$; and C, A. tenuissima, scale $=53 \mu \mathrm{M}$. 
(Andersen and Thrane 1996). Species clustered within the infectoria clade are not known to produce toxins, although recent data have shown that isolates of $A$. infectoria from wheat produce more metabolites than previously observed (Oviedo et al. 2013).

The lack of stability in morphological characteristics among environments continues to make Alternaria taxonomy a challenge. Because of this confusion, species are often grouped together and evaluated as one. Grouping of all small-spored Alternaria spp. as A. alternata can mask subtle differences in species biology and epidemiology. For example, this will limit proper determination of host ranges for many of these species. A. arbusti and A. arborescens may be examples of fungi isolated on potato that have been previously misidentified as A. alternata. A. arbusti was first isolated from Pyrus pyrifolia and has also been isolated from Rainier cherry in Washington (Simmons 1993), which are grown in close proximity to potato in the Columbia Basin of Washington. In this study, A. arborescens was isolated more frequently than A. alternata from lesions on potato, which is the predominant small-spored Alternaria sp. isolated from potato (Boiteux and Reifschneider 1994; Droby et al. 1984b; Hausladen 2006; van der Waals et al. 2011). A. arborescens has been isolated from potato in Iran (Ardestani et al. 2010); however, it has not yet been reported on potato in the United States.

More species of Alternaria pathogens have been shown to be associated with potato in the U.S. Northwest than previously thought. Accurate identification methods are necessary so that a more thorough understanding of Alternaria epidemiology can be obtained. Although morphology is capable of distinguishing among morphologically distinct small-spored Alternaria taxa, molecular techniques have helped to rapidly and accurately distinguish among morphologically similar species when appropriate loci are used. The results of these molecular assays for species designation will facilitate future research, such as population genetics studies, that may reveal other discrete characteristics of the species as related to disease spread or management.

\section{Acknowledgments}

We thank M. Pavek, G. Vandemark, F. Dugan, and D. Wheeler for critical reviews of the manuscript; D. Drader, M. Hubbard, and J. Miller for providing potato leaf samples; and the Washington State Potato Commission for partial funding support.

\section{Literature Cited}

Andersen, B., and Thrane, U. 1996. Differentiation of Alternaria infectoria and Alternaria alternata based on morphology, metabolite profiles, and cultural characteristics. Can. J. Microbiol. 42:685-689.

Andrew, M., Peever, T., and Pryor, B. 2009. An expanded multilocus phylogeny does not resolve morphological species within the small-spored Alternaria species complex. Mycologia 101:95-109.

Ardestani, S. T., Sharifnabi, B., Zare, R., and Moghadam, A. A. 2010. New Alternaria species associated with potato leaf spot in various potato growing region of Iran. Iran. J. Plant Pathol. 45:83-86.

Berbee, M., Pirseyedi, M., and Hubbard, S. 1999. Cochliobolus phylogenetics and the origin of known, highly virulent pathogens, inferred from ITS and glyceraldehyde-3-phosphate dehydrogenase gene sequences. Mycologia 91: 964-977.

Boiteux, L., and Reifschneider, F. 1994. Potato early blight caused by Alternaria alternata in Brazil. Plant Dis. 78:101.

Dobinson, K. F. 1995. Genetic transformation of the vascular wilt fungus Verticillium dahliae. Can. J. Bot. 73:710-715.

Droby, S., Dinoor, A., Prusky, D., and Barkai-Golan, R. 1984a. Pathogenicity of Alternaria alternata on potato in Israel. Phytopathology 74:537-542.

Droby, S., Prusky, D., Dinoor, A., and Barkai-Golan, R. 1984b. Alternaria alternata: A new pathogen on stored potatoes. Plant Dis. 68:160-161.
Gilchrist, D. G., Bostock, R. M., and Wang, H. 1995. Sphingosine-related mycotoxins in plant and animal diseases. Can. J. Bot. 73:459-467.

Hausladen, H. 2006. Potato early blight (Alternaria ssp.) in Germany. PPO Spec. Rep. No. 11:313.

Hong, S. G., Cramer, R. A., Lawrence, C. B., and Pryor, B. M. 2005. Alt a 1 allergen homologs from Alternaria and related taxa: Analysis of phylogenetic content and secondary structure. Fungal Genet. Biol. 42:119-129.

Kirk, W., and Wharton, P. 2012. Brown leaf spot. Mich. Ext. Bull. E-3182. Department of Plant, Soil and Microbial Science, Michigan State University, East Lansing, MI

Lawrence, D. P., Gannibal, P. B., Dugan, F. M., and Pryor, B. M. 2014 Characterization of Alternaria isolates from the infectoria species-group and a new taxon from Arrhenatherum, Pseudoalternaria arrhenatheria sp. nov. Mycol. Prog. 13:257-276.

Lawrence, D. P., Gannibal, P. B., Peever, T. L., and Pryor, B. M. 2013. The sections of Alternaria: Formalizing species-group concepts. Mycologia 105 530-546.

Lee, S., Taylor, J., Innis, M., Gelfand, D., Sninsky, J., and White, T. 1990. PCR Protocols: A Guide to Methods and Applications. Academic Press, New York.

Misaghi, I., Grogan, R., Duniway, J., and Kimble, K. 1978. Influence of environment and culture media on spore morphology of Alternaria alternata. Phytopathology 68:29-34.

Mohan, S., Davis, J., Sorensen, L., and Schneider, A. 1992. Infection of aerial parts of potato plants by Colletotrichum coccodes and its effects on premature vine death and yield. Am. Potato J. 69:547-559.

Nolte, P. 2008. Brown spot and black pit of potato: The other early blight. Am Veg. Grow. 56:32-33

Oviedo, M. S., Sturm, M. E., Reynoso, M. M., Chulze, S. N., and Ramirez, M. L. 2013. Toxigenic profile and AFLP variability of Alternaria alternata and Alternaria infectoria occurring on wheat. Braz. J. Microbiol. 44:447-455.

Peever, T., Canihos, Y., Olsen, L., Ibanez, A., Liu, Y.-C., and Timmer, L. 1999. Population genetic structure and host specificity of Alternaria spp. causing brown spot of Minneola tangelo and rough lemon in Florida. Phytopathology 89:851-860.

Peever, T., Su, G., Carpenter-Boggs, L., and Timmer, L. 2004. Molecular systematics of citrus-associated Alternaria species. Mycologia 96:119-134.

Pryor, B. M., and Bigelow, D. M. 2003. Molecular characterization of Embellisia and Nimbya species and their relationship to Alternaria, Ulocladium and Stemphylium. Mycologia 95:1141-1154.

Pryor, B. M., and Gilbertson, R. L. 2000. Molecular phylogenetic relationships amongst Alternaria species and related fungi based upon analysis of nuclear ITS and mt SSU rDNA sequences. Mycol. Res. 104:1312-1321.

Pryor, B. M., and Michailides, T. J. 2002. Morphological, pathogenic, and molecular characterization of Alternaria isolates associated with Alternaria late blight of pistachio. Phytopathology 92:406-416.

Rodrigues, T., Berbee, M., Simmons, E., Cardoso, C., Reis, A., Maffia, L., and Mizubuti, E. 2010. First report of Alternaria tomatophila and A. grandis causing early blight on tomato and potato in Brazil. New Dis Rep 22:28.

Rotem, J. 1994. The Genus Alternaria: Biology, Epidemiology, and Pathogenicity. American Phytopathological Society, St. Paul, MN.

Simmons, E. G. 1992. Alternaria taxonomy: Current status, viewpoint, and challenge. Pages 1-36 in: Alternaria: Biology, Plant Diseases, and Metabolites. J. Chelkowski and A. Visconti, eds. Elsevier Science Publishers, New York.

Simmons, E. G. 1993. Alternaria themes and variations (63-72). Mycotaxon 48 91-107.

Simmons, E. G. 2007. Alternaria: An Identification Manual. CBS Fungal Biodiversity Centre, Utrecht, The Netherlands.

Stevenson, W., Kirk, W., and Atallah, Z. 2007. Pages 209-222 in: Management of Foliar Disease, Early Blight, Late Blight and White Mold. Potato Health Management. American Phytopathological Society, St Paul, MN.

Taralova, E. H., Schlecht, J., Barnard, K., and Pryor, B. M. 2011. Modelling and visualizing morphology in the fungus Alternaria. Fungal Biol. 115:1163-1173.

Thomma, B. P. 2003. Alternaria spp.: From general saprophyte to specific parasite. Mol. Plant Pathol. 4:225-236.

Tymon, L. S., and Johnson, D. A. 2014. Fungicide resistance of two species of Alternaria from potato in the Columbia Basin of Washington. Plant Dis. 98: $1648-1653$

van der Waals, J. E., Pitsi, B., Marais, C., and Wairuri, C. K. 2011. First report of Alternaria alternata causing leaf blight of potatoes in South Africa. Plant Dis. $95: 363$. 